\title{
DEVELOPMENT OF A MINING MACHINE SEAT MODEL
}

\author{
Paulina Działak $^{1}$, Jacek Karliński ${ }^{1}$, Eugeniusz Rusiński ${ }^{1}$ \\ 1 Wroclaw University of Science and Technology, Department of Mechanical Engineering, \\ Łukasiewicza 7/9, 50-371, Wrocław, Poland. \\ mail: paulina.dzialak@pwr.edu.pl, jacek.karlinski@pwr.edu.pl, eugeniusz.rusinski@pwr.edu.pl
}

\section{Introduction}

The authors have endeavoured to present the process of the mining machine seat modeling. The relevant data necessary to obtain the proper model where received from two experimental tests. Photogrammetrical survey was used to accomplish the geometry of the seat. To obtain the characteristic of the mounting and the material data for the cushion seat foam, the measurements on the universal testing machine were taken. The model was inspired by the Maximo seat of Grammer company (Fig. 1).

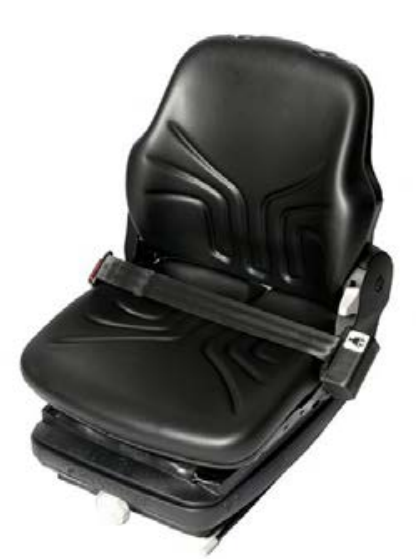

Fig. 1. Seat of the mining machine operator subjected to the measurements [1].

The main methods that lead to develop a complete computational model for the further simulation testing of the safety of a mining machine operators [2] were presented in the paper.

\section{Photogrammetric methods}

The geometrical model of the mining machine operator seat was created using an optical system TRITOP and the ATOS Compact Scan device. TRITOP is a portable system, measuring 3D coordinates quickly and precisely. It records points and their orientation in space at quasi-static conditions. Then, the measurement is transformed into the coordinate system of the component.
The shape of a cushion of the seat was determined by the TRITOP device (Fig. 2).

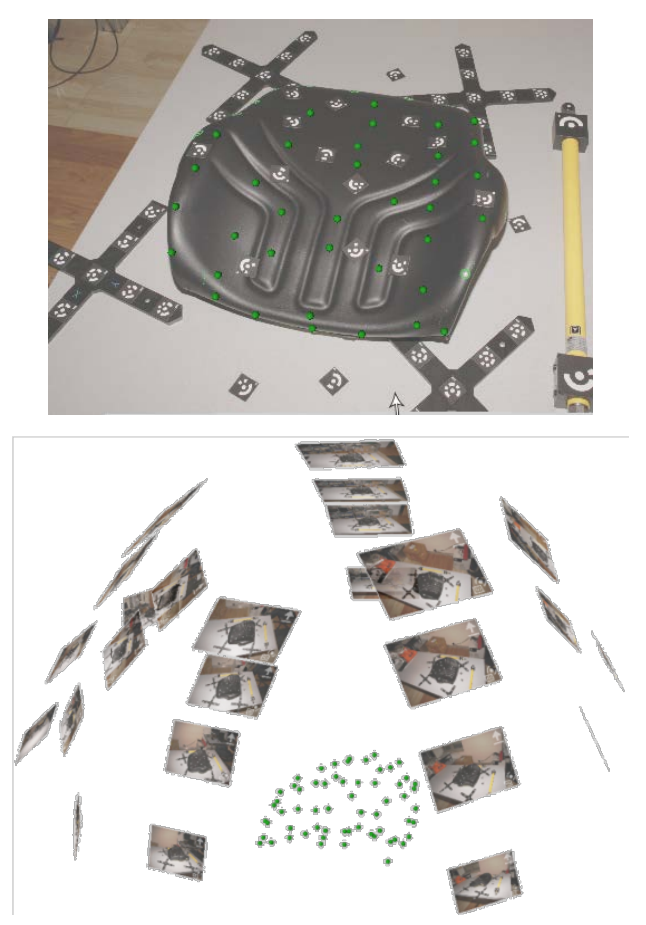

Fig. 2. Seat base during the TRITOP measurements (above) and received images with resulting point cloud (below).

The recreation of the geometry was accomplished by the cloud of points received from the system. The geometry of the cushions of a base and the backrest of the operator's seat were obtained by creating surfaces on the spread points.

ATOS on the other hand is an optical 3D scanner based on fringe projection, delivering accurate and traceable 3D coordinates. The technology ensure an adaptability for 3dimensional measuring of the complex components. Using this method provides a high accuracy and results in complete data even if the shape of the object is complicated and the surface is shiny [3].

The geometry of the seat back obtained by the ATOS scanning device is presented in figure 3. 

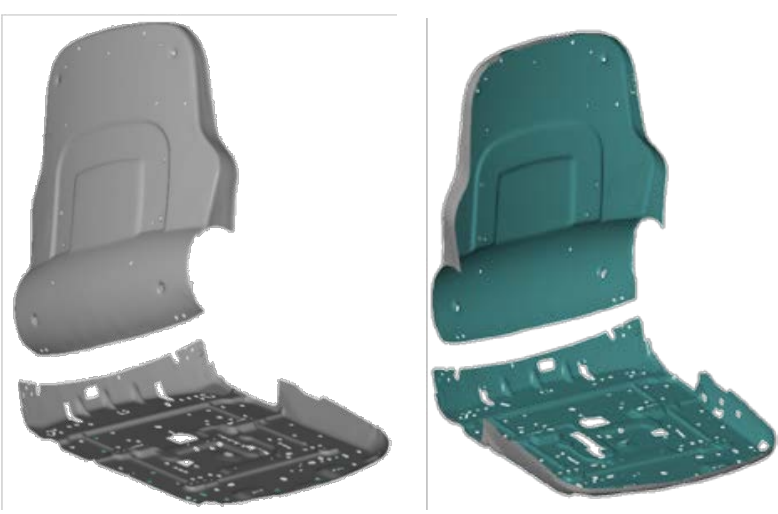

Fig. 3. Geometry of a seat frame (ATOS).

\section{Universal testing machine analysis}

The stiffness and strength of the seat mounting were examined experimentally on the universal testing machine Zwick Z030 [4]. The measurements enabled the authors to design a simplified model of the mounting (Fig. 4).

The material data for the cushion seat foam were also obtained empirically from the testing machine.

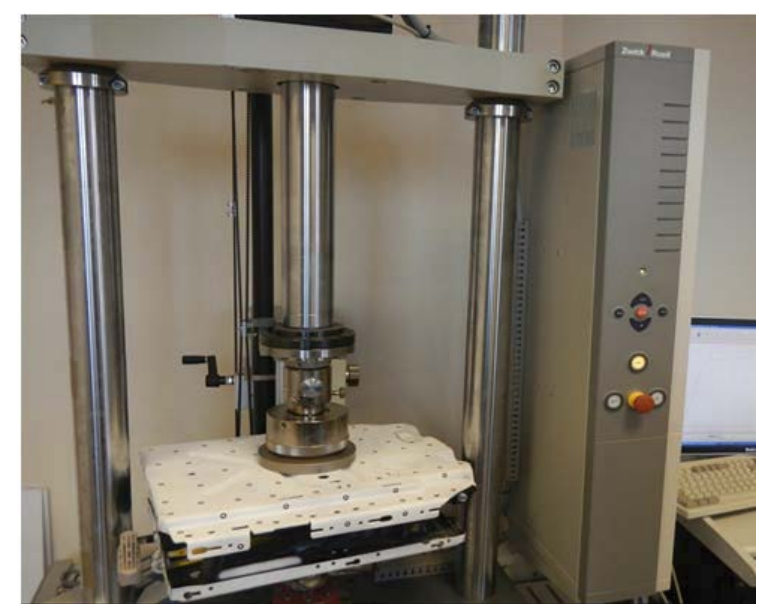

Fig. 4. Measurements of a seat mounting taken on the universal testing device.

\section{Numerical model}

The discrete model was generated, basing on the obtained geometrical shape of the seat and the parameters of the mounting and the material data.

The complete numerical model of the seat of a mining machine operator, achieved by the described process is presented in figure 5 .

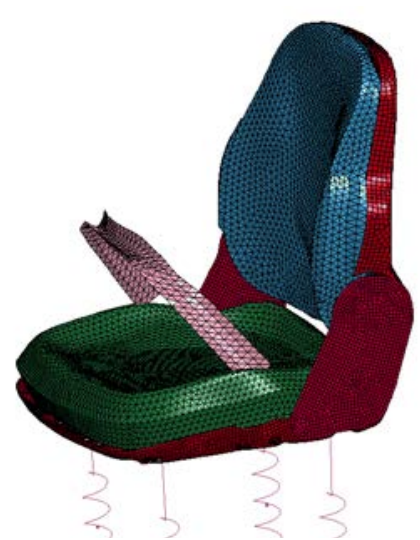

Fig. 5. Final numerical model of the seat.

\section{Remarks}

- The authors attempted to create the numerical equivalent of the seat structure of the mining machine operator.

- To reproduce the geometry two different photogrammetric methods were used.

- To obtain the characteristics of the seat mounting and the material data, the measurements on the universal testing machine were also performed.

\section{Acknowledgements}

Research is co-financed by the National Science Centre Poland within the framework of the project The assessment of the seismic phenomena effects in the aspect of the operator safety no. 2015/17/N/ST8/01212.

\section{References}

[1] https://www.grammer.com/en/productsmarkets/seating-systems/constructionmachinery/bm-maximo.html, 09.2016.

[2] Derlukiewicz, D., Karliński, J., Iluk, A., The operator protective structures testing for mining machines, Solid State Phenomena, Vol. 165, 2010. pp. 256-261.

[3] http://www.gom.com/metrologysystems/atos/atoscompactscan.html, 01.2017.

[4] Xu, J., Lu, L. F., Yang, H. W., Wang, J. N., Zhang, Y., Experimental Research on Relationship of Load and Displacement of Universal Testing Machine, Advanced Materials Research, Vols. 250-253, 2011. pp. 1582-1587. 